\title{
A PHENOMENON OF RECIPROCITY IN THE UNIVERSAL STEENROD ALGEBRA
}

\author{
LUCIANO LOMONACO
}

\begin{abstract}
ABSTRACr. In this paper we compute the cohomology algebra of certain subalgebras $L_{r}$ and certain quotients $K_{s}$ of the mod 2 universal Steenrod algebra $Q$, the algebra of cohomology operations for $H_{\infty}$-ring spectra (see [M]). We prove that

$$
\operatorname{Ext}_{L_{r}}\left(F_{2}, F_{2}\right) \cong K_{-k+1}, \quad \operatorname{Ext}_{K_{s}}\left(F_{2}, F_{2}\right) \cong L_{-s+1}
$$

with $r, s$ integers and $r \leq 1, s \geq 0$. We also observe that some of the algebras $L_{r}, K_{s}$ are well known objects in stable homotopy theory and in fact our computation generalizes the fact that $H^{*}\left(A_{L}\right) \cong \Lambda^{\text {opp }}$ and $H^{*}\left(\Lambda^{\text {opp }}\right) \cong A_{L}$ (see, for instance, $[\mathrm{P}]$ ). Here $A_{L}$ is the Steenrod algebra for simplicial restricted Lie algebras and $\Lambda$ is the $E_{1}$-term of the Adams spectral sequence discovered in [B-S].
\end{abstract}

\section{INTRODUCTION}

We recall that in [M] J. P. May introduced, for each prime $p$, an algebra $\mathscr{A}_{p}$ generated by symbols $P^{s} \quad(s \in \mathbb{Z})$ subject to a generalized version of Adem relations. We call $\mathscr{A}_{p}$ the mod $p$ universal Steenrod algebra because, as shown in [M], it is the algebra of cohomology operations in the category of $H_{\infty}$-ring spectra, and most of the algebras of operations (in homology and cohomology) which arise in algebraic topology can be obtained from $\mathscr{A}_{p}$ as subalgebras or subquotients. For example, the algebra $\Lambda^{\text {opp }}$ is contained in $\mathscr{A}_{p}$, the Steenrod algebra $A$ is a quotient of $\mathscr{A}_{p}$, and the Dyer-Lashof algebra $\mathscr{R}$ is a subquotient of $\mathscr{A}_{p}$. We focus our attention on the case $p=2$ and write $Q$ for the mod 2 universal Steenrod algebra.

In [L] the algebra $Q$ has been studied, and an invariant theoretical description of $Q$ has been given, generalizing some of the methods and ideas of $\mathrm{W}$. Singer [S]. In the present paper we would like to study the behaviour of the cohomology algebras of some subalgebras and quotients of $Q$. As we will make an extensive use of Priddy's results on Koszul algebras (see [P]), a section of this paper will be devoted to a brief summary of the definitions and results that will be needed in the sequel.

We find that there are two families of homogeneous Koszul algebras $\left\{L_{r}\right\}_{r \leq 1}$ and $\left\{K_{s}\right\}_{s \geq 0}$ with the following properties. For each integer $r \leq 1, L_{r}$ is a

Received by the editors August 17, 1989 and, in revised form, March 15, 1990.

1991 Mathematics Subject Classification. Primary 55S99.

Key words and phrases. Universal Steenrod algebra, Koszul algebras. 
subalgebra of $Q$, and for each integer $s \geq 0, K_{s}$ is a quotient of $Q$. We compute the cohomology of all such algebras and prove the following

Theorem. (i) For each $r \leq 1, H\left(L_{r}\right) \cong K_{-r+1}$

(ii) For each $s \geq 0, H\left(K_{s}\right) \cong L_{-s+1}$.

Some of these algebras are well known. For example $L_{0} \cong \Lambda^{\text {opp }}$ and $K_{1} \cong A_{L}$ where $A_{L}$ is the Steenrod algebra for simplicial restricted Lie algebras. Therefore, the above theorem generalizes a result of Priddy (see [P] and Corollary 4.2(i) below).

\section{Koszul algebras}

Let $F$ be a field, $\mathscr{I}$ a subset of $\mathbb{Z}$, and $\left\{x_{i}\right\}_{i \in \mathcal{I}}$ a set of symbols. We write $T$ for the free associative $F$-algebra over $\left\{x_{i}\right\}_{i \in \mathcal{I}} . T$ is a bigraded object: the first grading is obtained by assigning length $k$ to the monomials of the form $x_{i_{1}} \cdots x_{i_{k}}\left(i_{1}, \ldots, i_{k}\right) \in \mathscr{I}$ (repetitions are allowed), and the second grading is given by the total degree of a monomial, where each generator $x_{h}$ is assigned degree $h . T$ is augmented by the natural projection $\varepsilon: T \rightarrow F$. Suppose now that $B$ is an augmented $F$-algebra. A presentation

$$
\pi: T \rightarrow B
$$

is an augmented epimorphism for a suitable free associative $F$-algebra $T$ onto $B$.

Definition 2.1. $B$ is a homogeneous pre-Koszul algebra if it admits a presentation $\pi$ such that the two-sided ideal $\operatorname{ker}(\pi)$ is generated by elements of the form

$$
\sum_{i} \beta_{i} x_{k_{i}} x_{h_{i}} \quad\left(\beta_{i} \in F\right)
$$

We set $b_{i}=\pi\left(x_{i}\right) .\left\{b_{i}\right\}_{i \in \mathcal{I}}$ is called a set of pre-Koszul generators for $B . \pi$ induces on $B$ the length grading of $T$. If we also assume that in (2) the integer $k_{i}+h_{i}$ is constant, $\pi$ also induces on $B$ the grading given by the total degree of monomials in $T . B$ is therefore bigraded. We assume $B$ is of finite type, i.e., finite dimensional in each bidegree. The cohomology algebra associated to $B$,

$$
H(B)=\operatorname{Ext}_{B}(F, F),
$$

is trigraded (by the homological degree first, and then by length and total degree).

Definition 2.2. A homogeneous pre-Koszul algebra $B$ is a homogeneous Koszul algebra if $H(B)$ is generated, as an algebra, by any $F$-vector space basis of monomials of $H^{1,1, *}(B)$, or equivalently if $H^{r, s, *}(B)=0$ unless $r=s$.

Let

$$
U=\bigcup_{n>0} \mathscr{I} \times \cdots \times \mathscr{I} \quad(n \text {-copies })
$$

be the set of multi-indices, and let $\mathscr{B}$ be an $F$-vector space basis of monomials for $B$. If $b_{i_{1}} \cdots b_{i_{k}}$ is a monomial, we write

$$
b_{I}=b_{i_{1}} \cdots b_{i_{k}} \text {, }
$$


where $I=\left(i_{1}, \ldots, i_{k}\right) \in U$, and we say that the multi-index $I$ is the label of the monomial $b_{I}$. Let $S=\left\{I \in U \mid b_{I} \in \mathscr{B}\right\}$. The pair $(\mathscr{B}, S)$ is called a labelled basis for $B$. If $B$ is a homogeneous Koszul algebra and $(\mathscr{B}, S)$ is a labelled basis for $B$, the generating relations for $B$ can be written as

$$
b_{h} b_{k}=\sum_{(i, j) \in S} f(h, k, i, j) b_{i} b_{j}, \quad(h, k) \in \mathscr{I} \times \mathscr{I}, f \in F .
$$

Let $\mathscr{B} *$ denote the dual basis of $\mathscr{B}$. If $b_{I} \in \mathscr{B}$, we write $\alpha(I)$ or $\alpha\left(i_{1}, \ldots, i_{k}\right)$ for its corresponding dual element, i.e.,

$$
\alpha(I) \in \operatorname{Hom}(B, F),
$$

and we have

$$
\left\langle\alpha(I), b_{J}\right\rangle= \begin{cases}1 & \text { if } J=I, \\ 0 & \text { if } J \in S-\{I\} .\end{cases}
$$

Let us write $\alpha_{i}$ for the cohomology class of the cocycle $[\alpha(i)]$ in the cobar construction.

The following theorem, due to Priddy [P], is very useful and easy to prove.

Theorem 2.3. With the notation used above, if $B$ is a homogeneous Koszul algebra, $(\mathscr{B}, S)$ is a labelled basis, and (3) represents the generating relations, then the cohomology algebra $H(B)$ is generated by the classes $\alpha_{i}, i \in \mathscr{I}$, subject to the following relations:

$$
\alpha_{i} \alpha_{j}=\sum_{(h, k) \notin S} f(h, k, i, j) \alpha_{h} \alpha_{k} \quad((i, j) \in S) .
$$

We remark that the set $U$ is totally ordered, by length first and then lexicographically.

Definition 2.4. A labelled basis $(\mathscr{B}, S)$ is a Poincaré-Birkhoff-Witt (PBW) basis if the following two conditions hold:

(i) If $I, J \in S$, then either $(I, J) \in S$ or else the label of each monomial appearing in the expression of $b_{I} b_{J}$ as a linear combination of elements of $\mathscr{B}$ is strictly greater than $(I, J)$.

(ii) Let $k>2$. Then $\left(i_{1}, \ldots, i_{k}\right) \in S$ if and only if for each $j<k$ we have $\left(i_{1}, \ldots, i_{j}\right) \in S,\left(i_{j+1}, \ldots, i_{k}\right) \in S$.

Here $(I, J)$ indicates the multi-index obtained by juxtaposing $J$ to $I$.

Theorem 2.5. If $B$ is a homogeneous pre-Koszul algebra and it admits a PBWbasis, then $B$ is a homogeneous Koszul algebra.

Theorem 2.6. If both $B$ and $H(B)$ are homogenous Koszul algebras, then

$$
H(H(B)) \cong B \text {. }
$$

For proofs of Theorems 2.5 and 2.6 , see [P].

\section{THE ALGEBRA $Q$ AND OTHER RELATED ALGEBRAS}

From now on we will only consider $F_{2}$-algebras. Here we are going to outline a short description of the mod 2 universal Steenrod algebra $Q$. For more details and an invariant theoretical description of $Q$, see [L].

Let $T$ be the free associative algebra on generators $\left\{x_{i}\right\}_{i \in \mathbb{Z}}$, and let $D: T \rightarrow$ $T$ be the derivation defined by setting $D\left(x_{i}\right)=x_{i-1}, i \in \mathbb{Z}$. We write $D^{j}$ for $D \circ \cdots \circ D$ ( $j$-copies), and $D^{0}$ for the identity map. 
Definition 3.1. We set $Q=T / I$, where $I$ is the two-sided ideal of $T$ generated by all the elements of the form $D^{j}\left(x_{2 i-1} x_{i}\right), j \geq 0, i \in \mathbb{Z}$.

Theorem 3.2 (see $[\mathrm{L}]) . Q$ can be presented by generators $x_{i}, i \in \mathbb{Z}$, and relations

$$
x_{2 k-1-n} x_{k}=\sum\left(\begin{array}{c}
n-1-j \\
j
\end{array}\right) x_{2 k-1-j} x_{k+j-n} \quad(n \geq 0, k \in \mathbb{Z})
$$

(which we call generalized Adem relations).

If $I=\left(i_{1}, \ldots, i_{n}\right), i_{j} \in \mathbb{Z}$, is a multi-index, we write $x_{I}$ instead of $x_{i_{1}} \cdots x_{i_{n}}$. We recall that the set of admissible monomials

$$
\mathscr{B}=\left\{x_{I} \mid n \geq 0, i_{j} \geq 2 i_{j+1} \text { for each } j=1, \ldots, n-1\right\}
$$

is a linear basis for $Q$.

Definition 3.3. For each $r \in \mathbb{Z}$ we let $L_{r}$ be the subalgebra of $Q$ generated by $x_{r}, x_{r-1}, x_{r-2}, \ldots$.

Proposition 3.4. For each $r \leq 1, L_{r}$ can be presented by generators $x_{r}, x_{r-1}$, $x_{r-2}, \ldots$ and relations

(6) $x_{2 k-1-n} x_{k}=\sum\left(\begin{array}{c}n-1-j \\ j\end{array}\right) x_{2 k-1-j} x_{k+j-n}$

$$
(n \geq 0, k \leq r, 2 k-1-n \leq r) .
$$

Proof. We observe that since $k \leq r \leq 1$, we have $2 k-1 \leq k$ and thus

$$
2 k-1-j \leq k \leq r \text { for each } j .
$$

Moreover, the binomial coefficient $\left(\begin{array}{c}n-1-j \\ j\end{array}\right)$ does not vanish only if $0 \leq 2 j \leq$ $n-1$. In particular, we have $j<n$, and therefore

$$
k+j-n<k \leq r \text { for each } j<n .
$$

Now we let $L_{r}^{\prime}$ be the algebra presented by generators $y_{i}, i \leq r$, and relations

(7) $y_{2 k-1-n} y_{k}=\sum\left(\begin{array}{c}n-1-j \\ j\end{array}\right) y_{2 k-1-j} y_{k+j-n}$

$$
(k, 2 k-1-n \leq r, n \geq 0) .
$$

We define a homomorphism $\gamma: L_{r}^{\prime} \rightarrow Q$ by setting $\gamma\left(y_{i}\right)=x_{i}, i \leq r$. Clearly $\operatorname{Im}(\gamma)=L_{r}$. Moreover, $\gamma$ is a monomorphism. In fact, if $y \in L_{r}^{\prime}$ is a polynomial expression of the $y_{i}$ 's and $x=\gamma(y)=0$ in $Q$, this means that we can apply generalized Adem relations to the inadmissible pairs $x_{a} x_{b}$ appearing in some of the monomials in $x$, and after applying finitely many such relations we find that $x=0$, in $Q$. All such relations are also available in $L_{r}^{\prime}$, so $y=0$ in $L_{r}^{\prime}$, i.e., $\gamma$ is a monomorphism and $L_{r}^{\prime} \cong L_{r}$.

Remark 3.5. For $r \geq 2$, Proposition 3.4 is false. For example, take $r=2$. In $Q$ we have

$$
x_{2} x_{2}=x_{3} x_{1}
$$

Hence

$$
x_{2} x_{2} x_{1}=x_{3} x_{1} x_{1}=0 \text { in } Q
$$


as $x_{1} x_{1}=0 . L_{2}$ is a subalgebra of $Q$, thus

$$
x_{2} x_{2} x_{1}=0 \text { in } L_{2}
$$

and it is not possible to obtain such a relation in $L_{2}$ by handling relations of the form (6) (with $r=2$ ), as in $L_{2}$ relation (8) is not available $\left(x_{3} \notin L_{2}\right)$.

In [BG] an algebra $\bar{\Lambda}$ was introduced. We look at the opposite of $\bar{\Lambda} . \bar{\Lambda}^{\text {opp }}$ is presented by generators $\lambda_{i}, i \geq-1$, and relations

$$
\lambda(p, q)=0, \quad p, q \geq 0,
$$

where

$$
\lambda(p, q)=\sum_{j \geq 0}\left(\begin{array}{l}
p \\
j
\end{array}\right) \lambda_{2 q+j-1} \lambda_{p+q-j-1} .
$$

The algebra $\Lambda^{\text {opp }}$ (the opposite of the algebra $\Lambda$ defined in [B-S]) is a subalgebra of $\bar{\Lambda}^{\mathrm{opp}}$ and is presented by generators $\lambda_{i}, i \geq 0$, and relations

$$
\lambda(p, q)=0, \quad p \geq 0, q>0 \text {. }
$$

Proposition 3.6. (i) $L_{1} \cong \bar{\Lambda}^{\mathrm{opp}}$.

(ii) $L_{0} \cong \Lambda^{\mathrm{opp}}$.

Proof. An isomorphism $\phi: \bar{\Lambda}^{\mathrm{opp}} \rightarrow L_{1}$ (which restricts to an isomorphism $\left.\Lambda^{\text {opp }} \rightarrow L_{0}\right)$ is given by setting $\phi\left(\lambda_{i}\right)=x_{-i}$. $\phi$ is well defined, as

$$
\phi(\lambda(p, q))=\sum\left(\begin{array}{l}
p \\
j
\end{array}\right) x_{-2 q-j+1} x_{-p-q+j+1}=D^{p}\left(x_{1-2 q} x_{1-q}\right) .
$$

The inverse of $\phi$ is also well defined, as it is easy to check.

Proposition 3.7. For each $r \leq 1, L_{r}$ is a homogeneous Koszul algebra.

Proof. By Proposition 3.4, for each $r \leq 1 \quad L_{r}$ is a homogeneous pre-Koszul algebra. Moreover, the subset $\mathscr{B}_{r} \subseteq \mathscr{B}$ consisting of all the admissible monomials $x_{I}$ with $x_{j} \leq r$ for each $j$ is a PBW-basis, as it is easy to check.

Remark 3.8. $Q$ fails to be a homogeneous Koszul algebra, because it is not of finite type.

For each $s \in \mathbb{Z}$, let us consider the two-sided ideal

$$
I(s)=\left(x_{s-1}, x_{s-2}, x_{s-3}, \ldots\right) \subseteq Q .
$$

Definition 3.9. For each $s \in \mathbb{Z}$ we define an algebra $K_{s}$ by setting

$$
K_{s}=Q / I(s) \text {. }
$$

Proposition 3.10. For each $s \geq 0, K_{s}$ is presented by generators $x_{s}, x_{s-1}$, $x_{s+2}, \ldots$ and relations of the form (5) with $k \geq s$ and $2 k-1-n \geq s$, where $a$ summand $x_{2 k-1-j} x_{k+j-n}$ in the RHS of (5) is taken to be zero if $k+j-n<s$.

Proof. Clearly $K_{s}$ is presented by generators $x_{i}, i \geq s$, and relations of the form (5), modulo $x_{a}=0$ if $a<s$. Therefore $K_{s}$ is presented by generators $x_{i}, i \geq s$, and relations of the form (5), with $2 k-1-n \geq s, k \geq s$, modulo 
$x_{a}=0$ if $a<s$, plus, possibly, relations of the form (5), with $2 k-1-n<s$ or $k<s$, modulo $x_{a}=0$ if $a<s$, having some nonvanishing summands on the RHS of (5). We want to show that these latter relations do not actually occur. In fact, if $k<s$, for each $j$ such that $\left(\begin{array}{c}n-1-j \\ j\end{array}\right) \neq 0$ we have $2 j \leq n-1$, hence $j<n$ and $k+j-n<k<s$, therefore each summand on the RHS of (5) vanishes in this case. On the other hand, if $2 k-1-n<s$, and we assume $k+j-n \geq s$ and $2 k-1-j \geq s$, we would have $k \geq s+n-j$, i.e., $2 k \geq 2 s+2 n-2 j$. But we know that, in order for $\left(\begin{array}{c}n-1-j \\ j\end{array}\right) \neq 0$, we must have $j \leq n-1-j$, i.e., $1 \leq n-2 j$. We would get

$$
2 k \geq 2 s+n(n-2 j) \geq 2 s+n+1
$$

and therefore

$$
2 k-1-n \geq 2 s \geq s \quad(\text { as } s \geq 0),
$$

a contradiction.

Let us define an algebra $\bar{A}$ by the presentation

$$
\begin{array}{r}
\bar{A}=\left\langle S q^{0}, S q^{1}, S q^{2}, \ldots\right| S q^{a} S q^{b}=\sum\left(\begin{array}{c}
b-1-j \\
a-2 j
\end{array}\right) S q^{a+b-j} S q^{j}, \\
a<2 b, a, b \geq 0\rangle .
\end{array}
$$

We observe that the Steenrod algebra $A$ can be obtained as a quotient of $\bar{A}$ by adding the extra relation $S q^{0}=1$. The Steenrod algebra for simplicial restricted Lie algebras $A_{L}$ can also be obtained as a quotient of $\bar{A}$ by adding the extra relation $S q^{0}=0$.

Proposition 3.11. (i) $K_{0} \cong \bar{A}$.

(ii) $K_{1} \cong A_{L}$.

Proof. Let us consider an Adem relation

$$
S q^{a} S q^{b}=\sum\left(\begin{array}{c}
b-1-j \\
a-2 j
\end{array}\right) S q^{a+b-j} S q^{j}, \quad a<2 b .
$$

As $a<2 b$, we can write $a=2 b-1-m$ for a suitable nonnegative integer $m$. (9) becomes

$$
S q^{2 b-1-m} S q^{b}=\sum\left(\begin{array}{c}
b-1-j \\
2 b-1-m-2 j
\end{array}\right) S q^{3 b-1-m-j} S q^{j}
$$

Now we notice that

$$
\left(\begin{array}{c}
b-1-j \\
2 b-1-m-2 j
\end{array}\right)=\left(\begin{array}{c}
b-1-j \\
m+j-b
\end{array}\right)
$$

and set $i=m+j-b$. We make the above substitution in (10) to get

$$
S q^{2 b-1-m} S q^{b}=\sum\left(\begin{array}{c}
m-1-i \\
i
\end{array}\right) S q^{2 b-1-i} S q^{b+i-m}
$$

After making this remark, it is easy to see that an isomorphism $\psi: \bar{A} \rightarrow K_{0}$ can be defined by setting $\psi\left(S q^{i}\right)=x_{i}$. Moreover, $\psi$ induces an isomorphism between $A_{L}$ and $K_{1}$. 
Proposition 3.12. For each $s \geq 0, K_{s}$ is a homogeneous Koszul algebra.

Proof. For each $s \geq 0, K_{s}$ is a homogeneous pre-Koszul algebra, because of Proposition 3.10. Moreover, the admissible monomials which do not involve generators $x_{i}$ with $i<s$ form a PBW-basis.

Remark 3.13. For $s<0$ such admissible monomials fail to form a basis, as they are not linearly independent. For example, take $s=-1$ and consider the relation

$$
x_{-2} x_{1}=x_{1} x_{-2}+x_{0} x_{-1} \text { in } Q,
$$

which we write as

$$
x_{-2} x_{1}+x_{1} x_{-2}=x_{0} x_{-1} \text { in } Q .
$$

As $x_{-2} \in I(-1)$, we have

$$
x_{0} x_{-1}=0 \text { in } K_{-1}
$$

although $x_{0} x_{-1}$ is admissible. Similarly, using the relation

$$
x_{-4} x_{1}=x_{1} x_{-4}+x_{0} x_{-3}+x_{1} x_{-2} \text { in } Q,
$$

we find that

$$
x_{0} x_{-3}+x_{1} x_{-2}=0 \text { in } K_{-3} \text {. }
$$

\section{Cоноmology computations}

Here we prove the result announced in the introduction.

Theorem 4.1. (i) For each $r \leq 1, H\left(L_{r}\right) \cong K_{-r+1}$.

(ii) For each $s \geq 0, H\left(K_{s}\right) \cong L_{-s+1}$.

Proof. We will prove (i) by a direct computation, using the machinery developed in §2. (ii) will follow from Theorem 2.6. By Theorem 2.3, we have that

$$
\begin{aligned}
H\left(L_{r}\right)=\left\langle\alpha_{i}, i \leq r\right| \alpha_{i} \alpha_{j}=\sum_{k<2 m} f(k, m, i, j) \alpha_{k} \alpha_{m}, & \\
& i \geq 2 j, i, j, k, m \leq r\rangle,
\end{aligned}
$$

where $f(k, m, i, j)$ is the coefficients of $x_{i} x_{j}$ in the admissible expression of $x_{k} x_{m}$ in $L_{r}$. As we have a relation for each monomial $\alpha_{i} \alpha_{j}$ with $i \geq 2 j$, we write such a relation as

$$
\alpha_{2 j+p} \alpha_{j}=\sum f(p, j, h) \alpha_{2 j+p-h} \alpha_{j+h},
$$

where $p \geq 0, j, 2 j+p \leq r$, and $2 j+p-h<2(j+h)$, i.e., $p-h<2 h$, i.e., $p<3 h$. Moreover, we must have $j+h, 2 j+p-h \leq r$, as $\alpha(2 j+p-h)$ and $\alpha(j+h)$ are required to be dual to elements of $L_{r}$. The scalar $f(p, j, h)$ is the coefficient of $x_{2 j+p} x_{j}$ in the admissible expression of $x_{2 j+p-h} x_{j+h}$ in $L_{r}$. We write $2 j+p-h$ as

$$
2 j+p-h=2(j+h)-1-n,
$$


where $n=3 h-1-p$. As $p<3 h$, we have $n \geq 0$. We now look at the Adem relation

$$
\begin{aligned}
x_{2 j+p-h} x_{j+h} & =x_{2(j+h)-1-n} x_{j+h} \\
& =\sum\left(\begin{array}{c}
n-1-t \\
t
\end{array}\right) x_{2(j+h)-1-t} x_{j+h+t-n}
\end{aligned}
$$

in $L_{r}$. We are looking for the coefficient of $x_{2 j+p} x_{j}$ in (12). In the RHS of (12) $x_{2 j+p} x_{j}$ appears when $h+t-n=0$. So its coefficient is

$$
\begin{aligned}
f(p, j, h) & =\left(\begin{array}{l}
h-1 \\
n-h
\end{array}\right)=\left(\begin{array}{c}
h-1 \\
3 h-1-p-h
\end{array}\right) \\
& =\left(\begin{array}{c}
h-1 \\
2 h-1-p
\end{array}\right)=\left(\begin{array}{l}
h-1 \\
p-h
\end{array}\right)
\end{aligned}
$$

(which does not depend on $j$ ). The generating relations for $H\left(L_{r}\right)$ are therefore of the form

$$
\alpha_{2 j+p} \alpha_{j}=\sum\left(\begin{array}{l}
h-1 \\
p-h
\end{array}\right) \alpha_{2 j+p-h} \alpha_{j+h},
$$

where we mean $\alpha_{q}=0$ if $q>r$. We now define a homomorphism $\omega$ : $H\left(L_{r}\right) \rightarrow K_{-r+1}$ by setting $\omega\left(\alpha_{i}\right)=x_{-i+1}$. The relation (13) is mapped to

$$
x_{-2 j-p+1} x_{-j+1}=\sum\left(\begin{array}{l}
h-1 \\
p-h
\end{array}\right) x_{-2 j-p+h+1} x_{-j-h+1}
$$

$$
\left(\bmod x_{q}=0 \text { if } q<-r+1\right) .
$$

If we set $a=-j+1$ and $b=p-h$, the above relation becomes

$$
x_{2 a-1-p} x_{a}=\sum\left(\begin{array}{c}
p-1-b \\
b
\end{array}\right) x_{2 a-1-b} x_{a+b-p}
$$

$$
\left(\bmod x_{q}=0 \text { if } q<-r+1\right),
$$

which is a relation in $K_{-r+1}$. Hence $\omega$ is well defined and, in a similar manner, we can check that the map $\bar{\omega}: K_{-r+1} \rightarrow H\left(L_{r}\right)$, which takes $x_{c}$ to $\alpha_{-c+1}$, is also a well-defined homomorphism. Clearly $\bar{\omega}$ is the inverse of $\omega$ and $\omega$ is an isomorphism.

As a consequence of the above theorem, using Propositions 3.5 and 3.11, we find the following

Corollary 4.2. (i) $H\left(A_{L}\right) \cong \Lambda^{\text {opp }} ; H\left(\Lambda^{\mathrm{opp}}\right) \cong A_{L}$.

(ii) $H(\bar{A}) \cong \bar{\Lambda}^{\mathrm{opp}} ; H\left(\bar{\Lambda}^{\mathrm{opp}}\right) \cong \bar{A}$.

Part (i) is the well-known result of Priddy mentioned in the introduction.

\section{REFERENCES}

[B-S] A. K. Bousfield, E. B. Curtis, D. M. Kan, D. G. Quillen, D. L. Rector, and J. W. Schlesinger, The mod $p$ lower central series and the Adams spectral sequence, Topology 5 (1966), 331-342.

[BG] E. H. Brown and S. Gitler, A spectrum whose cohomology is a certain cyclic module over the Steenrod algebra, Topology 12 (1973), 283-295.

[L] L. Lomonaco, Dickson invariants and the universal Steenrod algebra, Rend. Circ. Mat. Palermo (2) 24 (1990), 429-444. 
[M] J. P. May, A general approach to Steenrod operations, Lecture Notes in Math., vol. 168, Springer-Verlag, 1970, pp. 153-231.

[P] S. B. Priddy, Koszul resolutions, Trans. Amer. Math. Soc. 152 (1970), 39-60.

[S] W. M. Singer, Invariant theory and the lambda algebra, Trans. Amer. Math. Soc. 280 (1983), 673-693.

Dipartimento di Matematica, Universita di Napoli, Napoli, Italia 